\title{
Competition between Superconductivity and Charge-density Wave Order in $\mathrm{Na}_{0.3} \mathrm{CoO}_{2} \cdot \mathbf{1 . 3 \mathrm { H } _ { 2 } \mathrm { O }}$
}

\author{
H. Fu • N. Oeschler • R.A. Fisher • N.E. Phillips • \\ J.E. Gordon · D.-H. Lee · M.-L. Foo · R.J. Cava
}

Received: 10 December 2008 / Accepted: 12 December 2008 / Published online: 10 January 2009

(C) The Author(s) 2009. This article is published with open access at Springerlink.com

\begin{abstract}
Three samples of $\mathrm{Na}_{0.3} \mathrm{CoO}_{2} \cdot 1.3 \mathrm{H}_{2} \mathrm{O}$ that differed in sample age showed different ordering: after three and five days, superconductivity with $T_{\mathrm{c}} \sim 4.5 \mathrm{~K}$ but different superconducting condensates; after 40 days, no superconductivity but charge-density wave order at $T_{\mathrm{CDW}} \sim 7 \mathrm{~K}$. A pair-breaking action that progresses with sample age and acts preferentially in one of two electron bands produces the changes in the superconducting condensate, and ultimately destroys the superconductivity. Theoretical calculations predicted the charge-density wave transition.
\end{abstract}

Keywords Cobaltate superconductor · Charge density wave order · Two electron bands

\section{Introduction}

Most of the structural and electronic properties thought to be important for superconductivity in the cuprates occur in $\mathrm{Na}_{0.3} \mathrm{CoO}_{2} \cdot 1.3 \mathrm{H}_{2} \mathrm{O}$, but the Co ions are in a magnetically frustrated triangular array, which may affect the

H. Fu · D.-H. Lee

Lawrence Berkeley National Laboratory and Department of Physics, University of California, Berkeley, CA 94720, USA

N. Oeschler · R.A. Fisher · N.E. Phillips ( $₫)$

Lawrence Berkeley National Laboratory and Department

of Chemistry, University of California, Berkeley, CA 94720, USA

e-mail: nephill@berkeley.edu

J.E. Gordon

Physics Department, Amherst College, Amherst, MA 01002, USA

M.-L. Foo · R.J. Cava

Physics Department, Princeton University, Princeton, NJ 08544, USA superconductivity. The comparison with the cuprates has generated considerable interest in the superconductivity of $\mathrm{Na}_{0.3} \mathrm{CoO}_{2} \cdot 1.3 \mathrm{H}_{2} \mathrm{O}$, for which the maximum $T_{\mathrm{c}}$ is $\sim 4.5 \mathrm{~K}$, but the strong sample dependence of the superconductingstate properties has precluded recognition of the intrinsic properties required for meaningful comparisons with theoretical predictions. In addition, there is a different transition near $7 \mathrm{~K}$ in some non-superconducting samples. There is also uncertainty in the nature of the band structure: most calculations show a large, cylindrical, zone-centered $a_{1 \mathrm{~g}}$ surface, but the existence of six small surrounding $e_{\mathrm{g}}^{\prime}$ pockets is controversial. Specific-heat data are more consistent with the presence of the $e_{\mathrm{g}}^{\prime}$ pockets than their absence $[1,2]$. There is additional evidence of their existence, but they are not seen in ARPES (see [2] for a review).

\section{Results and Discussion}

Samples 1, 2, and 3 were prepared as described elsewhere [3]; they were kept at room temperature with a small excess of $\mathrm{H}_{2} \mathrm{O}$ (to promote thermal contact) for 40,3 , and 5 days, respectively, before the specific heat was measured. The conduction-electron contribution to the specific heat $\left(C_{\mathrm{e}}\right)$ for Samples 2 and 3 is shown in Fig. 1. $C_{\mathrm{e}} / T$ does not extrapolate to 0 at $T=0$, but to $\gamma_{\mathrm{r}}$, a measure of the pair breaking [1,2]. For Samples 2 and 3 (at 3 and 5 days) $\gamma_{\mathrm{r}}$ is 40 and $70 \%$ of $\gamma_{\mathrm{n}}$, the coefficient of the normalstate $C_{\mathrm{e}}$. The contribution to $C_{\mathrm{e}}$ associated with the superconducting condensate is different, varying as $T^{2}$ and $\exp \left(-T^{-1}\right)$ for Samples 2 and 3, respectively. The quantity $C_{\mathrm{e}}^{\prime} \equiv\left(C_{\mathrm{e}}-\gamma_{\mathrm{r}} T\right) \gamma_{\mathrm{n}} /\left(\gamma_{\mathrm{n}}-\gamma_{\mathrm{r}}\right)$, which is a useful and reasonable approximation to $C_{\mathrm{e}}$ for one mole of superconducting condensate [2], i.e., in the absence of pair breaking, is shown 
in Fig. 2 where it is compared with BCS theory in the weakcoupling limit. The deviations from BCS theory are particularly conspicuous for Sample 2: At the lowest $T, C_{\mathrm{e}}$ is more than two orders of magnitude greater than the BCS value, characteristic of an energy gap smaller than the BCS gap; for $T / T_{\mathrm{c}} \geq 0.5$ the curvature of $C_{\mathrm{e}}^{\prime} / T$ is characteristic of a gap larger than the BCS gap. These deviations are almost the same as those for $\mathrm{MgB}_{2}$, for which they are associated with two electron bands with different superconducting-state energy gaps [4]. For Sample 3 the low- $T$ deviations are less conspicuous, but the high- $T$ deviations associated with a large gap are more pronounced. The comparison with $\mathrm{MgB}_{2}$ suggests two gaps in $\mathrm{Na}_{0.3} \mathrm{CoO}_{2} \cdot 1.3 \mathrm{H}_{2} \mathrm{O}$, and two-gap, $\alpha$ model [5] fits, similar to those for $\mathrm{MgB}_{2}$ [4], are included in Fig. 2. The two-gap fits give the values (shown in Fig. 2)

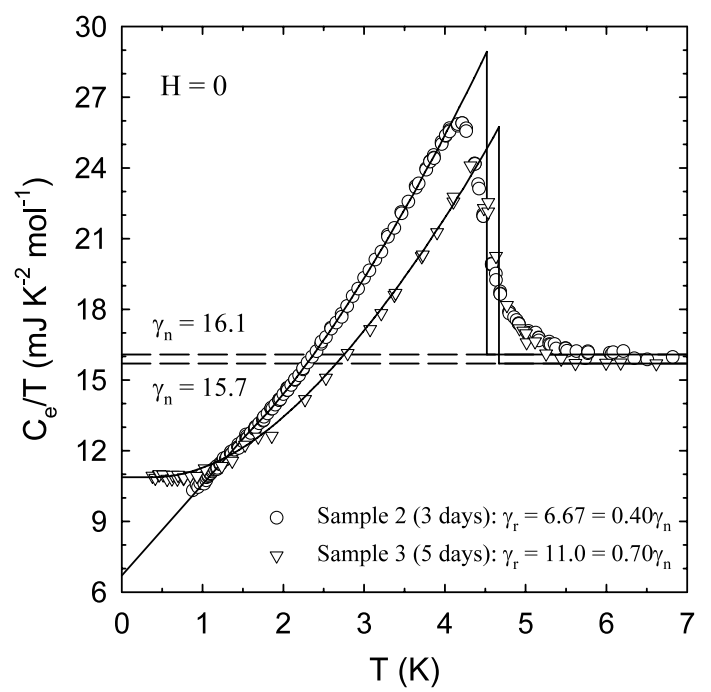

Fig. 1 The electron specific heat of Samples 2 and 3. The solid lines are two-gap fits to the experimental points that correspond to an energy gap with nodes for Sample 2, but without nodes for Sample 3 of the 0 -K gap, $\Delta_{i}(0)$, as $\alpha_{i} \equiv \Delta_{i}(0) / \mathrm{k}_{\mathrm{B}} T_{\mathrm{c}}$, and the contribution of the band to the superconducting condensate, as a fraction of the total normal-state density of states, $\gamma_{i s} / \gamma_{\mathrm{n}}$, for each band, $i=1,2$.

The specific-heat results provide an understanding of the evolution of the superconductivity and its eventual disappearance with increasing sample age: the pair breaking increases with increasing sample age, $40 \%$ at 3 days, $70 \%$ at 5 days, and $100 \%$ (no superconductivity) at 40 days. It acts preferentially in the band with the small gap: the Sample 2 to Sample 3 reduction in the small-gap band contribution to the superconducting condensate $\left(\gamma_{2 \mathrm{~s}} / \gamma_{\mathrm{n}}\right.$ changes from 0.27 to 0.06 ) is substantially greater than in the large-gap band $\left(\gamma_{1 \mathrm{~s}} / \gamma_{\mathrm{n}}\right.$ changes from 0.33 to 0.24$)$. Neutron diffraction measurements [6] complement these results and support this interpretation: they show that samples that are not initially superconducting become superconducting, $T_{\mathrm{c}}$ passes through a maximum, and superconductivity disappears. This process is accompanied by an increase in the concentration of $\mathrm{O}$ vacancies, the obvious candidates for the pair-breaking centers, all on a time scale similar to that of the changes in our samples. The plausibility of this interpretation is also supported by the approximate inverse relation between the increases in the pair breaking and the energy gaps, which measures the strength of the pairing interaction.

Weak electron pairing in the $a_{1 \mathrm{~g}}$ band (see below) suggests the identification of the small-gap band as the $a_{1 \mathrm{~g}}$ band. Extrapolation of the $\gamma_{i s} / \gamma_{\mathrm{n}}$ to zero pair breaking $\left(\gamma_{\mathrm{r}} / \gamma_{\mathrm{n}}=0\right)$ provides an estimate of the relative contributions of the two bands to the total normal-state density of states [2]: $56 \%$ for the small-gap, $a_{1 \mathrm{~g}}$ band, and $44 \%$ for the large-gap, $e_{\mathrm{g}}^{\prime}$, band.

As shown in Fig. 3, the 7-K specific-heat anomaly in Sample 1 and the 4.5-K anomaly in Sample 2 are similar in zero field. However, a field of $\mu_{0} H=9 \mathrm{~T}$ eliminates
Fig. 2 The electron specific heat of Samples 2 and 3 normalized to one mole of superconducting condensate (see text). The curves through the points are two-gap fits, as in Fig. 1, but here the fit for Sample 2 is for a gap without nodes, which fits the actual experimental data equally well. The light curves are the contributions of each band
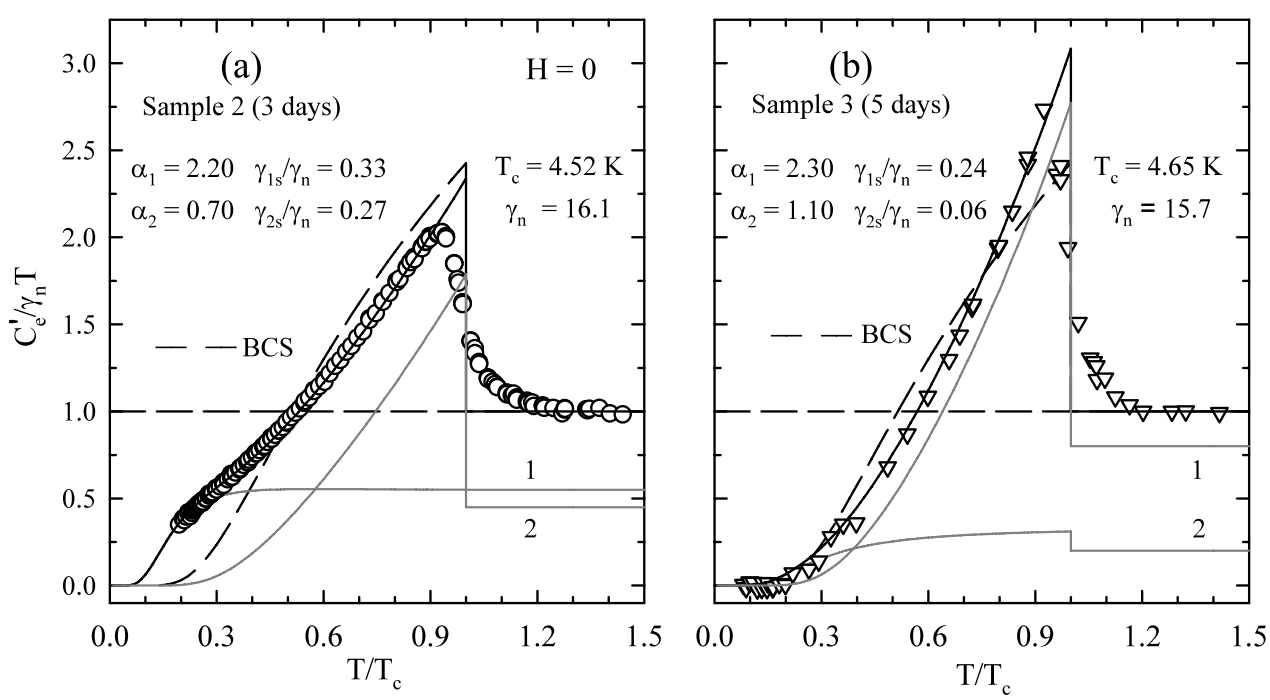
Fig. 3 The electron specific heat for Samples 2 and 1, showing superconductivity and CDW order, respectively. For Sample 2 the curve through the zero-field points is the two-gap fit shown in Fig. 1; for Sample 1 it is a mean-field fit described in the text

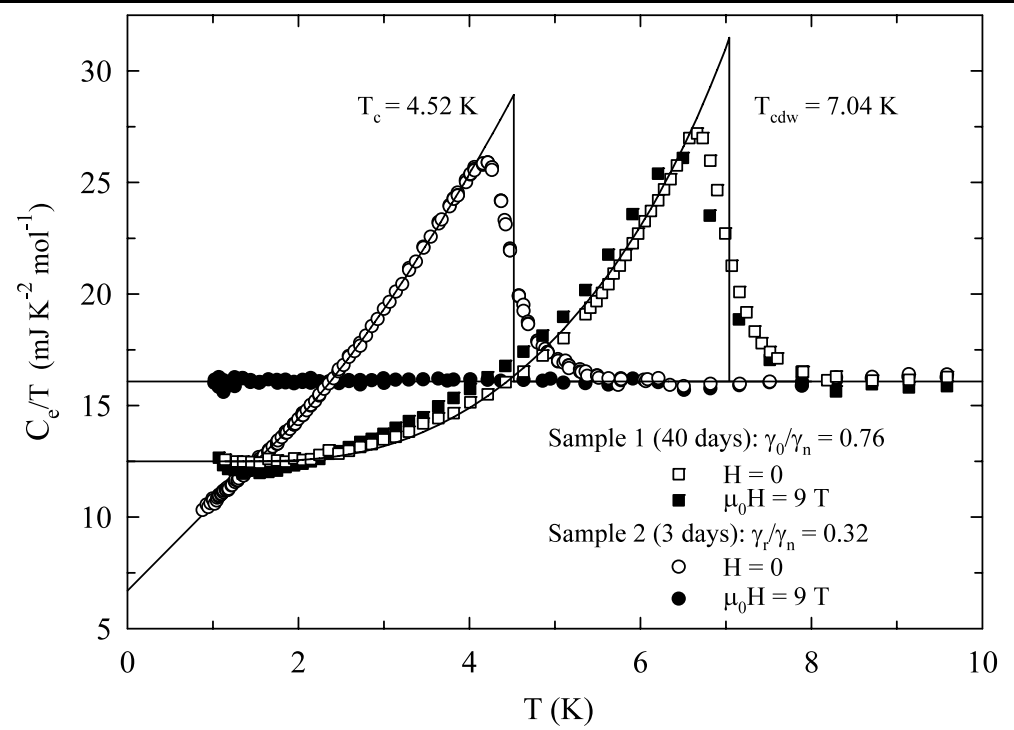

the Sample-2 anomaly, consistent with the transition to the superconducting state, but has essentially no effect on the Sample-1 anomaly, consistent with a transition to a chargedensity wave (CDW) state.

The experimental discovery of the 7-K transition was anticipated by a theoretical calculation (H. Fu, D.-H. Lee) that predicted CDW order. This was a numerical one-loop renormalization group calculation [7, 8], which used a discretization of a band structure [9] derived from ARPES results $[10,11]$ that showed only the $a_{1 \mathrm{~g}}$ Fermi surface. The initial (bare) electron-electron interaction potential is that of a $90 \mathrm{meV}$ on-site, and a $30 \mathrm{meV}$ nearest-neighbor, Hubbard interaction on a triangular lattice. Renormalization group flow strengthens negative interactions connecting wave vectors on opposite sides of an almost-nested Fermi surface, which can drive a $\sqrt{3} \times \sqrt{3}$ CDW ordering. This CDWpromoting interaction is the dominant feature that grows under renormalization group flow. There is evidence of a weak pairing interaction, but it is overwhelmed by the chargedensity wave interaction.

A mean-field fit to the specific heat in the vicinity of the $\mathrm{CDW}$ ordering (see Fig. 3) gives $T_{\mathrm{CDW}}=7.04 \mathrm{~K}$ and $\Delta(0) / \mathrm{k}_{\mathrm{B}} T_{\mathrm{CDW}}=2.85$. The high- $T$ and low- $T$ coefficients of $C_{\mathrm{e}} / T$ are $\gamma_{\mathrm{n}}=16.4 \mathrm{~mJ} \mathrm{~K}^{-2} \mathrm{~mol}^{-1}$, and $\gamma_{0}=$ $12.5 \mathrm{~mJ} \mathrm{~K}^{-2} \mathrm{~mol}^{-1}$, respectively. By this measure the CDW develops over $24 \%$ of the total Fermi surface, or, if the $a_{1 \mathrm{~g}}$ surface is $56 \%$ of the Fermi surface, over $55 \%$ of the $a_{1 \mathrm{~g}}$ surface. In any case, the development of the CDW is apparently limited by defects, presumably the same $\mathrm{O}$ vacancies that are the likely pair-breaking centers for Cooper pairs in the superconducting samples. Since the CDW and superconducting condensation energies are similar and $T_{\mathrm{CDW}}>T_{\mathrm{c}}$, it is not clear why CDW ordering does not occur in all samples.

\section{Conclusions}

In superconducting samples, pair breaking, probably by $\mathrm{O}$ vacancies, progresses with sample age, shifting the contributions of two electron bands to the superconducting condensate and eventually destroying the superconductivity. CDW order is predicted theoretically, and is observed in a sample that is not superconducting.

Acknowledgements The work at LBNL was supported by the Director, Office of Science, Office of Basic Energy Sciences, of the US Department of Energy, under Contract No. DE-AC02-05CH11231; at Princeton, by NSF grant DMR-0213706 and a DOE-BES grant DEFG02-98-ER45706. N.O. was supported, in part, by the DAAD.

Open Access This article is distributed under the terms of the Creative Commons Attribution Noncommercial License which permits any noncommercial use, distribution, and reproduction in any medium, provided the original author(s) and source are credited.

\section{References}

1. Oeschler, N., Fisher, R.A., Phillips, N.E., Gordon, J.E., Foo, M.-L., Cava, R.J.: Europhys. Lett. 82, 47011 (2008)

2. Oeschler, N., Fisher, R.A., Phillips, N.E., Gordon, J.E., Foo, M.-L., Cava, R.J.: Phys. Rev. B 78, 054528 (2008)

3. Schaak, R.E., Klimczuk, T., Foo, M.-L., Cava, R.J.: Nature 424, 527 (2003)

4. Fisher, R.A., Li, G., Lashley, J.C., Bouquet, F., Phillips, N.E., Hinks, D.G., Jorgensen, J.D., Crabtree, G.W.: Physica C 385, 180 (2003)

5. Padamsee, H., Neighbor, J.E., Schiffman, C.A.: J. Low Temp. Phys. 12, 387 (1973)

6. Barnes, P.W., Avdeev, M., Jorgensen, J.D., Hinks, D.G., Claus, H., Short, S.: Phys. Rev. B 72, 134515 (2005)

7. Honerkamp, C., Salmhofer, M., Furukawa, N., Rice, T.M.: Phys. Rev. B 63, 135109 (2001)

8. Fu, H.C., Honerkamp, C., Lee, D.-H.: Europhys. Lett. 75, 146 (2006) 
9. Zhou, S., Gao, M., Ding, H., Lee, P.A., Wang, Z.: Phys. Rev. Lett. 94, 206401 (2005)

10. Hasan, M.Z., Chuang, Y.D., Qian, D., Li, Y.W., Kong, Y., Kuprin, A.P., Fedorov, A.V., Kimmerling, R., Rotenberg, E., Rossnagel, K., Hussain, H., Koh, H., Rogado, N.S., Foo, M.L., Cava, R.J.: Phys. Rev. Lett. 92, 246402 (2004)
11. Yang, H.-B., Wang, S.-C., Sekharan, A.K.P., Matsui, H., Souma, S., Sato, T., Takahashi, T., Takeuchi, T., Campuzano, J.C., Jin, R., Sales, B.C., Mandrus, D., Wang, Z., Ding, H.: Phys. Rev. Lett. 92, 246403 (2004) 